\title{
Nanoscale
}

\section{Detection of stiff nanoparticles within cellular structures by contact resonance atomic force microscopy subsurface nanomechanical imaging}

\begin{tabular}{|c|c|}
\hline Journal: & Nanoscale \\
\hline Manuscript ID & Draft \\
\hline Article Type: & Paper \\
\hline Date Submitted by the Author: & $\mathrm{n} / \mathrm{a}$ \\
\hline Complete List of Authors: & $\begin{array}{l}\text { Reggente, Melania; Sapienza University of Rome, Department of Basic and } \\
\text { Applied Sciences for Engineering (BASE) } \\
\text { Passeri, Daniele; Universita' degli studi di Roma, La Sapienza, Basic and } \\
\text { Applied Sciences for Engineering } \\
\text { Angeloni, Livia; Sapienza University of Rome, Department of Basic and } \\
\text { Applied Sciences for Engineering (BASE) } \\
\text { Scaramuzzo, Francesca; Universita degli Studi di Roma La Sapienza } \\
\text { Barteri, Mario; Universita degli Studi di Roma La Sapienza } \\
\text { De Angelis, Francesca; Sapienza University of Rome } \\
\text { Persiconi, Irene; Universita degli Studi di Roma La Sapienza Dipartimento } \\
\text { di Biologia e Biotecnologie Charles Darwin } \\
\text { De Stefano, Maria Egle; Universita degli Studi di Roma La Sapienza } \\
\text { Dipartimento di Biologia e Biotecnologie Charles Darwin } \\
\text { Rossi, Marco; Sapienza University of Rome, Department of Basic and } \\
\text { Applied Sciences for Engineering (BASE); Sapienza University of Rome, } \\
\text { Center for Nanotechnologies applied to the Engineering (CNIS) }\end{array}$ \\
\hline \multicolumn{2}{|c|}{$\begin{array}{l}\text { Note: The following files were submitted by the author for peer review, but cannot be converted to } \\
\text { PDF. You must view these files (e.g. movies) online. }\end{array}$} \\
\hline \multicolumn{2}{|c|}{ Reggente_NPsCells_RSCtemplate_SUBMITTED.tex } \\
\hline
\end{tabular}


Dear Editor,

As the corresponding author and also on behalf of the other authors, I would like to the manuscript "Detection of stiff nanoparticles within cellular structures by contact resonance atomic force microscopy subsurface nanomechanical imaging" by M. Reggente, D. Passeri, L. Angeloni, F. A. Scaramuzzo, M. Barteri, F. De Angelis, I. Persiconi, M. E. De Stefano, and M. Rossi, to be considered for publication in Nanoscale.

The manuscript, which reports new research and results and is not under submission to other journals, describes for the first time the use of an advanced atomic force microscopy (AFM) based technique, i.e., contact resonance AFM (CR-AFM), for the imaging of subsurface agglomerate of nanoparticles internalized into microglia cells. The group of J. Killgore from NIST reported recently the use of the CR-AFM to detect nanoparticles buried under the surface of polymers in ad hoc realized reference samples. In our present work, for the first time, we extend this technique to the analysis of cells internalizing nanomaterials.

We thank you for your kind attention and we hope that the proposed paper will be considered for publication on Nanoscale.

Yours Faithfully,

Daniele Passeri 


\title{
Journal Name
}

\section{ARTICLE TYPE}

Cite this: DOI: $10.1039 / x x x x x x x x x x$

\section{Detection of stiff nanoparticles within cellular struc- tures by contact resonance atomic force microscopy subsurface nanomechanical imaging}

\author{
Melania Reggente, ${ }^{a, b}$ Daniele Passeri, ${ }^{* a}$ Livia Angeloni, ${ }^{a, c}$ Francesca Anna \\ Scaramuzzo, ${ }^{a}$ Mario Barteri, ${ }^{d}$ Francesca De Angelis, ${ }^{e, f}$ Irene Persiconi, ${ }^{g}$ Maria \\ Egle De Stefano, ${ }^{g}$ and Marco Rossi ${ }^{a, h}$
}

Received Date

Accepted Date

DOI: 10.1039/xxxxxxxxxx

www.rsc.org/journalname

Detecting stiff nanoparticles buried in soft biological matrices by atomic force microscopy (AFM) based techniques represents a new frontier in the field of scanning probe microscopies, originally developed as surface characterization methods. Here we report the detection of stiff (magnetic) nanoparticles (NPs) internalized in cells by using contact resonance AFM (CR-AFM) employed as a nondestructive subsurface characterization tool. Magnetite $\left(\mathrm{Fe}_{3} \mathrm{O}_{4}\right) \mathrm{NPs}$ were internalized in microglial cells from cerebral cortices of mouse embryos of 18 days by phagocytosis. Nanomechanical imaging of cells were recorded by detecting the contact resonance frequencies (CRFs) of an AFM cantilever held in contact with the sample. Agglomerates of NPs internalized in cells were visualized on basis of the local increase in the contact stiffness with respect to the surrounding biological matrix. A second AFM-based technique for nanomechanical imaging, magnetic force microscopy, and optical microscopy were used to confirm CR-AFM results. Thus, CR-AFM was demonstrated as a promising technique for subsurface imaging of nanomateri-

\footnotetext{
*E-mail: daniele.passeri@uniroma1.it

a Department of Basic and Applied Sciences for Engineering, SAPIENZA University of Rome, Rome, Italy.

${ }^{b}$ Institute of Physics and Chemistry of Materials of Strasbourg, UMR 7504-CNRS, Strasbourg University, Strasbourg, France.

${ }^{c}$ Lab. for Biomaterials and Bioengineering (CRC-I), Dept. Min-Met-Materials Eng. \& University Hospital Research Center, Laval University, Quebec City, Canada.

${ }^{d}$ Department of Chemistry, SAPIENZA University of Rome, Rome, Italy.

e Department of Anatomy, Histology, Forensic Medicine and Orthopaedics, SAPIENZA University of Rome, Rome, Italy.

${ }^{f}$ Center for Life Nano Science@Sapienza, Istituto Italiano di Tecnologia, Rome, Italy.

${ }^{g}$ Department of Biology and Biotechnology 'Charles Darwin', SAPIENZA University of Rome, Laboratory affiliated to Istituto Pasteur-Fondazione Cenci Bolognetti, Italy.

${ }^{h}$ Research Center for Nanotechnology applied to Engineering of SAPIENZA University of Rome (CNIS), Rome, Italy.
}

als in biological samples.

The capability of detecting nanoparticles (NPs) buried in soft biological samples represents one of the main challenges in bionanoscience, as it would allow the monitoring of the interactions between cells and NPs at the nanoscale. Developing innovative and nondestructive imaging techniques for the visualization of NPs embodied into cells with nanometer resolution is a challenging task of primary importance in several applications, from drug delivery to nanotoxicology ${ }^{1-4}$. The most common techniques currently used to analyze intracellular structures are based on optical, confocal and electron (both scanning and transmission) microscopies, which nevertheless have limited resolution and/or are destructive techniques that require several steps for the sample preparation, such as dehydration, fixation, metallization, or cross section cutting ${ }^{5-8}$. Even though atomic force microscopy (AFM) was originally developed as a surface characterization platform ${ }^{9}$, it potentially represents a useful alternative to develop nondestructive subsurface imaging. In AFM a sharp probe, i.e., the tip at the free-end of the cantilever, interacts with the surface of the investigated sample. The cantilever static deflection (in contact mode) or the amplitude of the oscillating cantilever (in tapping mode) are measured to reconstruct a map of the surface heights, i.e., the sample topography. Furthermore, since the probe physically interacts with the surface sample, different types of interaction forces can be monitored. Thus, several techniques have been developed to map local magnetic ${ }^{10}$, mechanical ${ }^{11}$, electrical ${ }^{12}$, thermal ${ }^{13}$ and chemical ${ }^{14}$ properties of the sample surface, with nanometer spatial resolution, together with the standard AFM topography. In these techniques, the probed tip-sample forces are the result of the interaction between the tip and a small volume of material below the surface of the sample with dimensions generally comparable to those of the tip. Therefore, they are sensitive 
not only to physical inhomogeneities on the surface, but also under the surface, within the sample probed volume, thus allowing the detection of buried nanostructures with properties different from those of the host matrix. For instance, magnetic force microscopy (MFM), electric force microscopy (EFM), and conductive AFM (C-AFM) derived techniques, such as dc-biased amplitude modulated AFM and Kelvin Probe AFM, have been successfully employed for the subsurface imaging of composite materials made of polymer matrices filled with $\mathrm{Fe}_{3} \mathrm{O}_{4}$ nanoparticles ${ }^{15}$ and carbon nanotubes ${ }^{16,17}$, or for the detection of magnetic NPs in biological matrices ${ }^{18-20}$. Since the presence of harder (softer) inclusions inside a softer (harder) matrix as well as voids beneath the sample surface cause local variations in the sample stiffness, subsurface imaging can be performed probing the different elastic properties of the host and guest systems. Thus, some of the different AFM based techniques which allow nanomechanical characterizations have been already used for the subsurface imaging of samples. Recently, Roduit et al. ${ }^{21,22}$ have proposed a method to analyze quasi static indentation curves acquired by AFM to obtain 3D stiffness tomography reconstructions. Moreover, the synergistic use of AFM and ultrasound testing resulted to be particularly effective for subsurface feature detection with nanometer lateral resolution. In particular, Yamanaka et al. used ultrasonic force microscopy (UFM) for the subsurface imaging on highly oriented pyrolytic graphite (HOPG) ${ }^{23}$. In addition to this, subsurface imaging of void defects in interconnections ${ }^{24}$ has been successfully performed by means of scanning near field ultrasound holography (SNFUH) ${ }^{25,26}$. To the best of our knowledge, this is the only AFM based technique which has been used for the visualization of single nanostructures, i.e., $\mathrm{SiO}_{2} \mathrm{NPs}$ and carbon nanohorns, embodied within a cell ${ }^{27,28}$. Finally, contact resonance AFM (CR-AFM) has been demonstrated to be a very versatile technique, which can be used to study materials in a wide range of mechanical properties, i.e., from stiff coatings and gemstones $^{29-32}$ to soft polymers ${ }^{33-35}$ and biological samples ${ }^{36}$. CR-AFM was used to study nanocomposite samples constituted by stiff nano-fillers into soft polymeric matrices, in which the presence of the nanomaterials was confirmed by the analysis of the variation of indentation modulus and its spatial distribution ${ }^{37-39}$. CR-AFM provided subsurface imaging by using ad hoc designed test samples, detecting voids generated by means of a focused ion beam (FIB) under the surface of Si single crystal ${ }^{40}$, as well as visualizing $\mathrm{SiO}_{2}$ NPs buried beneath a smooth and thin polystyrene film $^{41}$. However, to the best of our knowledge, CR-AFM has never been used to detect NPs internalized in cells.

Here we report, for the first time, the use of CR-AFM for the detection of stiff NPs embodied in cells. Because of their high phagocytic activity, microglia cells were used to produce model samples of cells engulfed with magnetic NPs, the presence of which was revealed by MFM.

Magnetite NPs were synthesized starting from Mohr salt $\left(\left(\mathrm{NH}_{4}\right)_{2} \mathrm{Fe}\left(\mathrm{SO}_{4}\right)_{2} \cdot 6 \mathrm{H}_{2} \mathrm{O}\right)$ and $\mathrm{Fe}_{2}\left(\mathrm{SO}_{4}\right)_{3}$ in ammonia solution containing $2 \%(\mathrm{w} / \mathrm{v})$ of polyvinylpyrrolidone (PVP), following the co-precipitation Massart's method ${ }^{42}$ that we modified as previously described ${ }^{20}$. Microglia cells were taken from cerebral cortices of 18 days-old mouse embryos, dissociating the tissue at

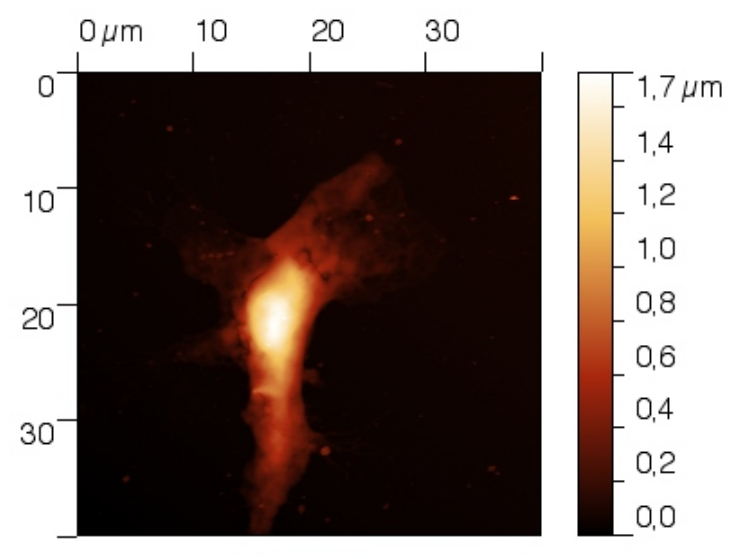

Fig. 1 Topography of a microglia cell on glass substrate recorded in tapping mode

$37^{\circ} \mathrm{C}$ with trypsin $0.1 \%$ and DNAse $(100 \mathrm{mg} / \mathrm{mL})$ in a suitable salt solution. A rather pure population of microglia (less than $2 \%$ of contamination) was obtained suspending them in Dulbecco's Modified Eagle Medium (DMEM) containing 10\% fetal bovine serum (FBS), placing them in a culture flask and leaving them in culture for 18 days. Subsequently, cells were made to adhere onto glass slides pretreated with poly-lysine $\left(10^{5}\right.$ cells/slide $)$. An example of microglia cell on glass substrate is shown in Fig. 1. $\mathrm{Fe}_{3} \mathrm{O}_{4}$ NPs were then added to the culture medium and internalized by endocytosis into the cytoplasm of microglia cells ${ }^{43}$. The process of phagocytosis required 5 hours of incubation in DMEM at $37^{\circ} \mathrm{C}$ under an atmosphere of air with $5 \% \mathrm{CO}_{2}$. After having removed the culture medium, the glass slide with the adherent cells was washed several times with a phosphate buffered saline (PBS) solution ( $\mathrm{pH}=7.4)$ to remove non-phagocytized NPs, and fixed for $15 \mathrm{~min}$ at room temperature in PBS with $4 \%$ formaldehyde. This fixation process, which implied the polymerization of cell membrane's proteins, altered the elastic properties of the cells making them stiffer, but still significantly softer than the NPs, allowing us to obtain a detectable contrast in CR-AFM images.

Optical microscopy images collected with $40 \times$ magnification (Fig. 2) highlighted the presence of NPs aggregates in close proximity to the cells, likely inside the cytoplasm.

Massive internalization of $\mathrm{Fe}_{3} \mathrm{O}_{4}$ NPs in microglia cells was assessed by MFM imaging, which confirmed the presence of a significant magnetic signal from labeled cells. An example is reported in Fig. 3, in which a standard MFM apparatus (Solver, NT-MDT, Russia) equipped with magnetic coated tips (MESP, Bruker Inc.) was used to acquire the topography of a microglia cell Fig.3a and the corresponding magnetic phase image Fig.3b. The dark contrast in the center of the MFM phase image indicates the presence of a massive agglomerate of magnetite NPs in the center of the cell (see for comparison Fig. 2a), although we made no attempts to improve the quality of the image by removing electrostatic artifacts from magnetic signal ${ }^{44,45}$.

CR-AFM is a contact mode based technique in which the back side of the sample is mounted on an ultrasonic transducer which excites out-of-plane oscillation of the sample surface. Spectra 


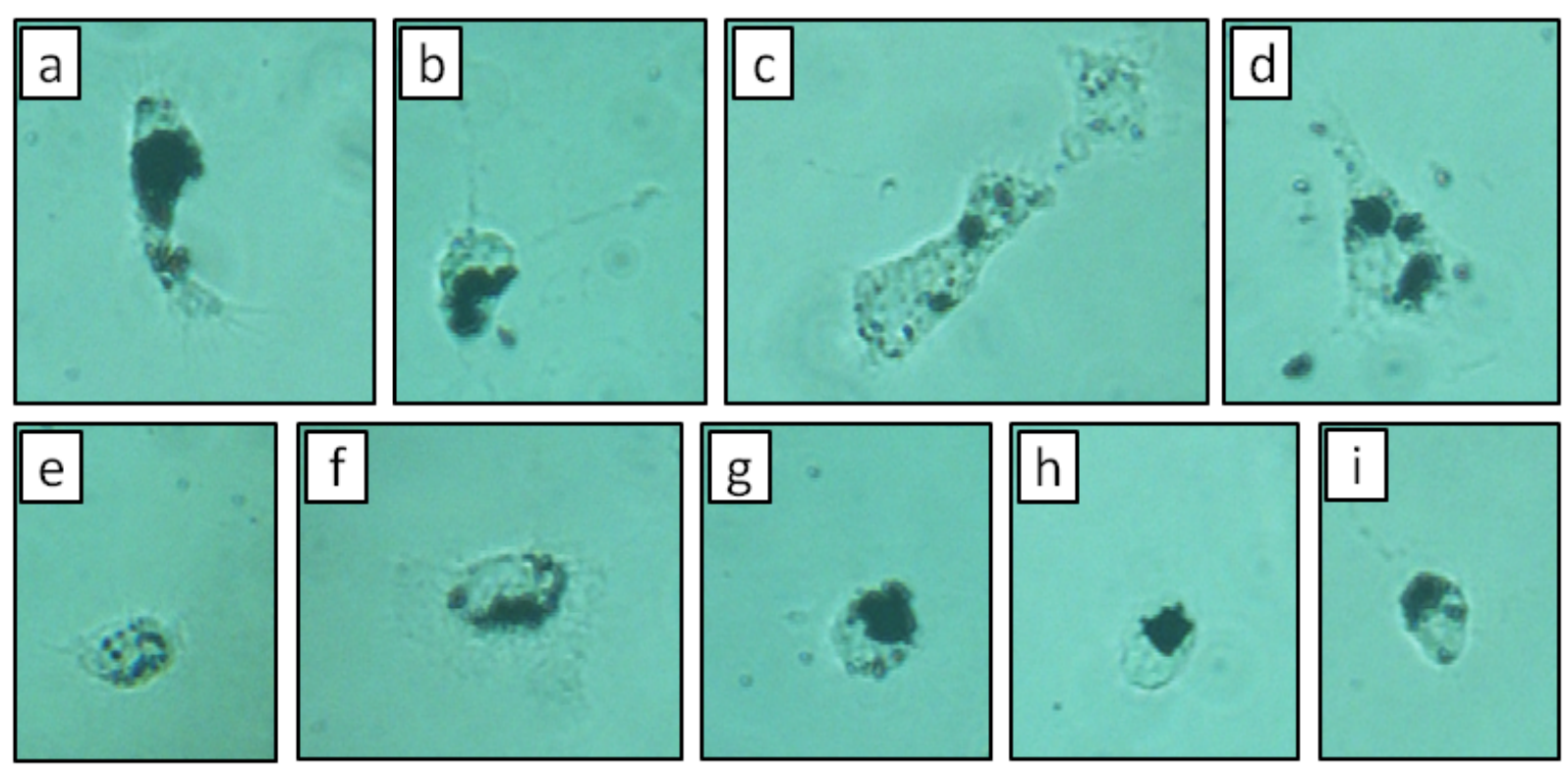

Fig. 2 Optical images (original magnification $40 \times$ ) of microglia cells incubated with magnetite NPs.

of the cantilever flexural vibrations are recorded and resonance frequencies of the cantilever-tip-sample system, namely the contact resonance frequencies (CRFs), are measured at each point of the scanned area and thus mapped on the sample surface simultaneously to the topography. The values of CRFs reflect the local elastic properties of the sample, i.e., the stiffer the sample, the higher the CRFs, and can be used to evaluate the sample indentation modulus. Therefore, CRFs maps can be considered as semi-quantitative nanomechanical images of the sample surface. Our AFM experimental setup (Solver, NT-MDT, Russia), the detailed description of which can be found elsewhere ${ }^{46}$, was equipped with commercial Si cantilevers (CSC17, MikroMasch, Estonia) chosen on the basis of their low spring constant (nominal value $k_{c}=0.18 \mathrm{~N} / \mathrm{m}$ ) to minimize surface modification and sample damaging during CR-AFM experiments.

Additionally, the chosen cantilevers are characterized by relatively low values of CRFs allowing their detection up to the sixth eigenmode. CR-AFM performed at higher eigenmodes has been demonstrated to have the maximum sensitivity to subsurface mechanical lack of homogeneity 41,47 . As expected, the values of CRFs measured on engulfed cells are significantly higher than those measured on the empty ones. This correspond to significantly larger values of normalized contact stiffness. Conversely, subsurface features in engulfed cells could be observed in CRFs images obtained using higher modes. In Fig. 4, the fifth eigenmode CRF maps of an empty cell (Fig. 4a) and of a cell engulfed with NPs (Fig. 4b) are shown. Images were obtained by searching the resonance in the range from $660 \mathrm{kHz}$ to $1100 \mathrm{kHz}$, i.e., between the fifth and the sixth eingenmodes of the free cantilever in air. As expected, CRFs values in correspondence of the cells are much lower than those in correspondence of the stiff substrate. In addition to this, CRFs values corresponding to empty cells are lower than those corresponding to engulfed ones. This can be attributed to a significant presence of $\mathrm{Fe}_{3} \mathrm{O}_{4} \mathrm{NPs}$ in labeled cells resulting in the increase of the effective elastic modulus of the cell. Moreover, in the case of empty cells (Fig. 4a), CRF values were constant within the cell and no areas corresponding to higher CRFs were detected. Conversely, large areas with higher CRFs were observed in the case of cells labeled with NPs (Fig. 4b). This suggests that $\mathrm{Fe}_{3} \mathrm{O}_{4} \mathrm{NPs}$ agglomerate within the structure of the cell, producing mechanical inhomogeneities detectable by CR-AFM nanomechanical imaging (see for comparison Fig. 2a, b, $g, h$, and i).

To deepen this phenomenon, several NPs labeled cells were investigated. Fig. 5a shows a labeled cell in which two distinct sub-micrometer spherical agglomerates are visible (see the detail in Fig. 5b, which can be compared to Fig. 2c, d, e and f). Overall, CR-AFM images revealed the presence of a single huge agglomerate like that in Fig. 4 or of one or more smaller agglomerates distributed in the cell volume like those in Fig. 5. In order to compare the capabilities of CR-AFM to detect stiff nanomaterials buried into cells to that of other AFM based techniques for the nanomechanical imaging, we performed HarmoniX ${ }^{\mathrm{TM}}$ (Dimension Icon, Bruker Inc.) on the same sample of microglia cells engulfed with NPs. HarmoniX ${ }^{\mathrm{TM}}$ takes advantage of the use of an out-ofaxis tip to set into torsional vibration a T-shaped cantilever during tapping ${ }^{48,49}$. The analysis of the cantilever torsional signal allows one to map the elastic modulus of polymers ${ }^{50-53}$ and biological materials ${ }^{54-56}$. However, we generally could not detect any subsurface structure in the engulfed microglia cells. Only in few cases we observed localized agglomerates, like in the case of the cell shown in Fig. 6 (see for comparison Fig. $2 g$ and h).

Without any relation with the topography (Fig. 6a), the stiffness map (Fig. 6b) shows an agglomerate (marked with the arrow), the stiffness of which is comparable with that of the glass substrate. The scarcity of agglomerates detected with HarmoniX ${ }^{\mathrm{TM}}$ can be rationalized considering that this technique probes the sample down to penetration depths smaller than CR- 


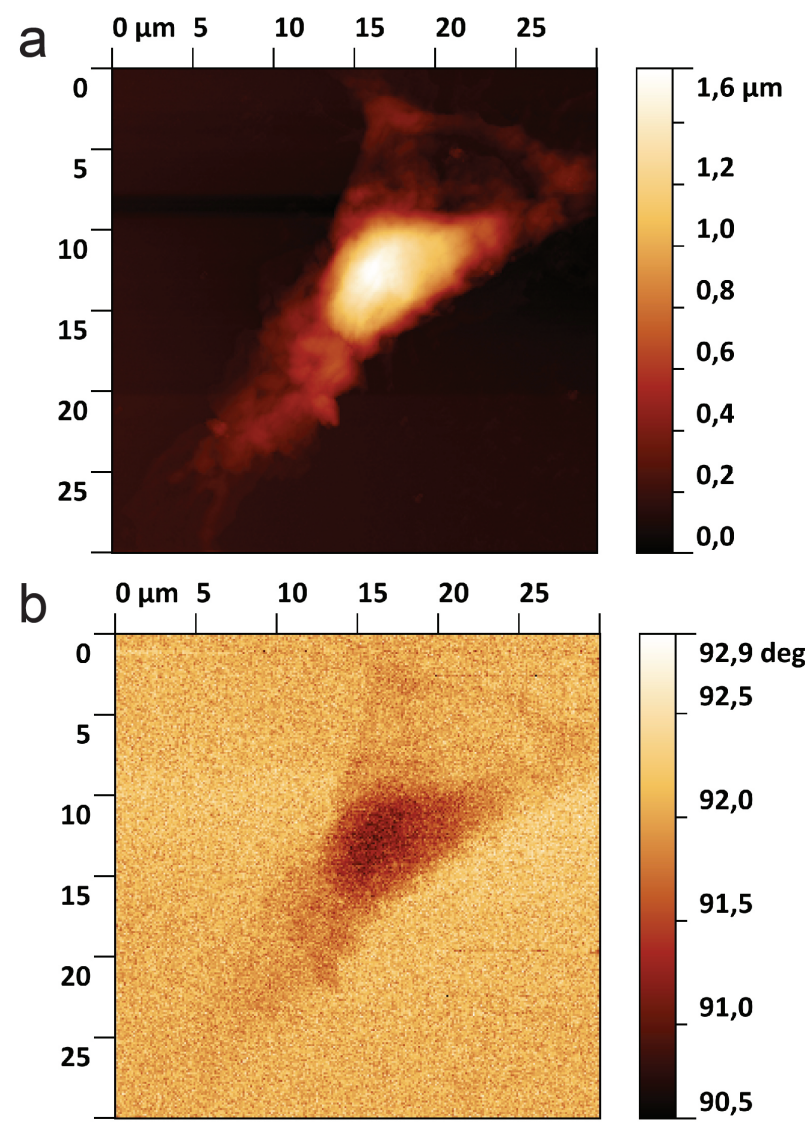

Fig. 3 (a) Topography of a microglia cell engulfed with NPs and (b) corresponding magnetic phase image obtained in magnetic force microscopy.

AFM. Indeed, we demonstrates that HarmoniX ${ }^{\mathrm{TM}}$ can be used on ultrathin soft samples, while we revealed the presence of stiff nanodiamonds particles only in the case they emerge from the surface or are buried immediately under the surface ${ }^{57}$. Due to the absence of contrast in the phase image, the agglomerate in Fig. 6 is likely to be buried under an ultrathin layer of biological material. Being more affected by the properties of the underlying layers, CR-AFM is more sensitive to buried stiff NPs. Notably, we could not detect isolated NPs using CR-AFM, but only massive agglomerates. This, however, does not necessarily indicate the absence of single NPs but may result from the presently limited resolution of CR-AFM.

Furthermore, the measured CRFs have been analyzed to calculate the apparent contact stiffness of each sample $k^{* 38}$. In particular, by considering the contact between the tip and the sample affected by both a vertical and a tangential spring (with spring constant defined by $k^{*}$ and $k_{\text {lat }}^{*}$, respectively), we simulated the $f_{5}$ values, for a range of $k^{*} / k_{c}$ between 1 and $2 \times 10^{4}$, by numerically solving the characteristic equation of the system ${ }^{38}$, considering the nominal dimensions of the cantilever supplied by the manufacturer and the beam inclined by an angle of 11 deg with respect to the sample surface. Values of $k^{*}$ calculated in this way for each sample are reported in Tab. 1 . As expected, the values of CRFs measured on engulfed cells significantly higher than those mea-
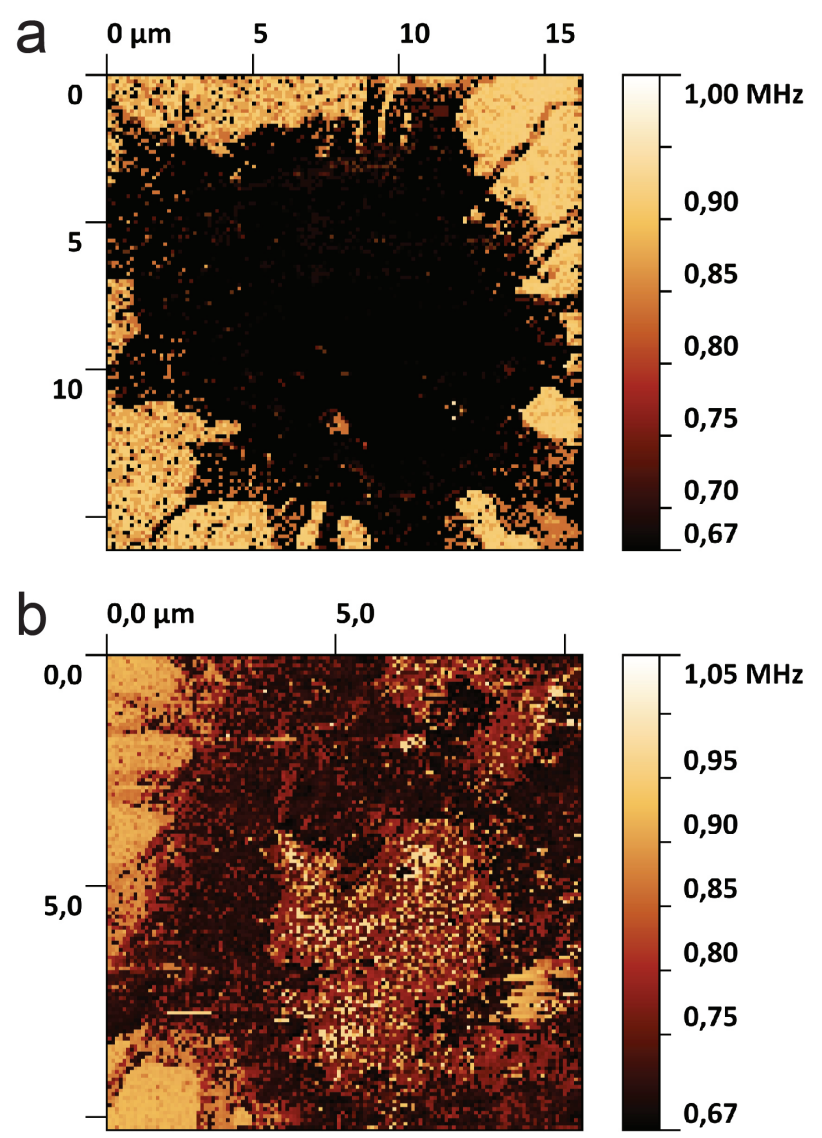

Fig. 4 (a) CRF map of an empty microglia cell; (b) CRF map of a microglia engulfed with NPs.

Table 1 Fifth eigenmode contact resonance frequencies $\left(f_{5}\right)$ and normalized contact stiffness $k^{*} / k_{c}$ values of empty and NP-engulfed microglia.

\begin{tabular}{ccc}
\hline Sample & $f_{5}(\mathrm{kHz})$ & $k^{*} / k_{c}$ \\
\hline empty cell & $670 \pm 6$ & $501 \pm 56$ \\
cell+NPs & $802 \pm 7$ & $1698 \pm 98$ \\
\hline
\end{tabular}

sured on the empty ones correspond to significantly larger values of normalized contact stiffness.

The calculated values of $k^{*} / k_{c}$ can be eventually used to estimate the depth at which the agglomerates are located. For this purpose, we assumed well-established models derived for the effective mechanical properties of layered materials during nanoindentation ${ }^{58}$, in the realistic approximation that the indentation modulus of the tip $\left(M_{t i p}\right)$ and of the $\mathrm{Fe}_{3} \mathrm{O}_{4}$ nanoparticles $\left(M_{N P s}\right)$ are much bigger than those of the microglia cells $\left(M_{\text {cell }}\right)$. The effective indentation modulus measured on the surface of NPsengulfed cells $\left(M_{\text {cell }+N P S}\right)$ in which NPs agglomerates are buried under a layer of cellular matrix with thickness $t$ can be evaluated as

$$
\frac{1}{M_{\text {cell }+N P s}}=\frac{1}{M_{\text {cell }}}(1-\exp (-\alpha t / a))
$$

in which $a$ is the radius of the contact area between the tip and the sample and $\alpha$ in a geometry-related parameter ${ }^{58}$. In order to obtain a rough estimation of $t$ from data reported in Tab. 1, exploit- 


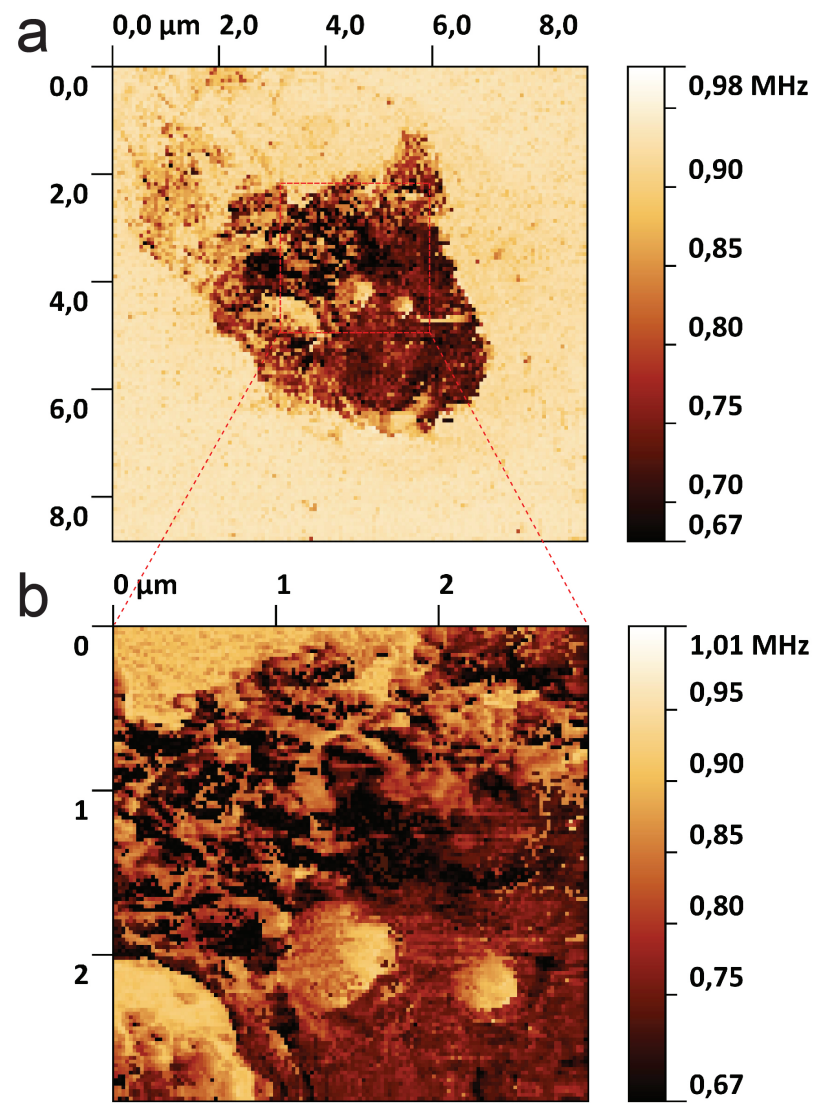

Fig. 5 (a) CRF map and (b) detail of a microglia cell detail engulfed with NPs.

ing the proportionality between the evaluated contact stiffness $k^{*}$ and the sample indentation modulus, we assumed a flat contact between the tip and the sample with radius $a=200 \mathrm{~nm}$, estimated by scanning electron microscopy analysis, and $\alpha=0.5^{58}$. Thus, Eq. (1) allows us to estimate that the agglomerates of NPs are approximately located at $t=100 \mathrm{~nm}$ under the sample surfaces. Even though these preliminary results do not provide the exact location of the nanoparticles, they demonstrate the feasibility of our approach for the detection of buried objects underneath the surface.

In conclusion, by detecting $\mathrm{Fe}_{3} \mathrm{O}_{4}$ NPs agglomerates into microglia cells, we demonstrated that CR-AFM can be a suitable technique for the subsurface imaging of stiff nanomaterials buried into biological samples. Although the resolution of the technique must be improved, CR-AFM is a promising tool for studying the fate of NPs into biological tissues.

\section{References}

1 R. D'Aquino, Chem. Eng. Prog., 2004, 100, 15S-17S

2 D. Peer, J. M. Karp, S. Hong, O. C. Farokhzad, R. Margalit and R. Langer, Nat. Nanotechnol., 2007, 2, 751-760.

3 L. Zhang, F. X. Gu, J. M. Chan, A. Z. Wang, R. S. Langer and O. C. Farokhzad, Clin. Pharmacol. Ther, 2008, 83, 761-769.

4 B. Chertok, B. A. Moffat, A. E. David, F. Yu, C. Bergemann, B. D. Ross and V. C. Yang, Biomaterials, 2008, 29, 487-496.

5 C.-C. Wang, C.-W. Lee, C.-Y. Huang, J.-Y. Lin, P.-K. Wei and C.-H. Lee, Appl. Optics, 2008, 47, 2458-2464.

6 E. A. Gibbs-Flournoy, P. A. Bromberg, T. P. Hofer, J. M. Samet and R. M. Zucker, Part. Fibre Toxicol., 2011, 8, 1. a
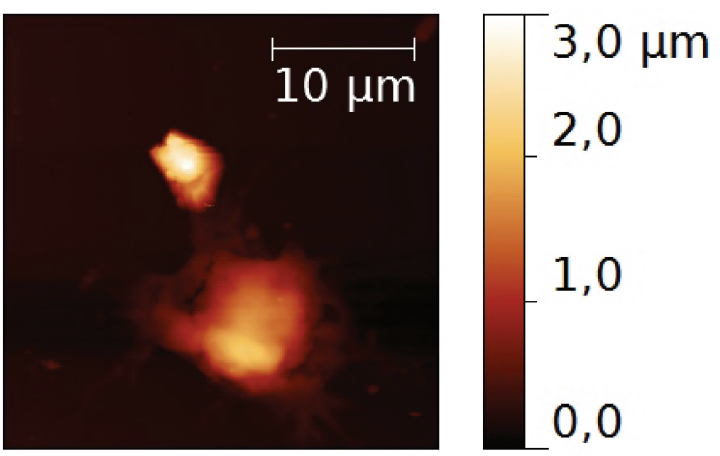

b

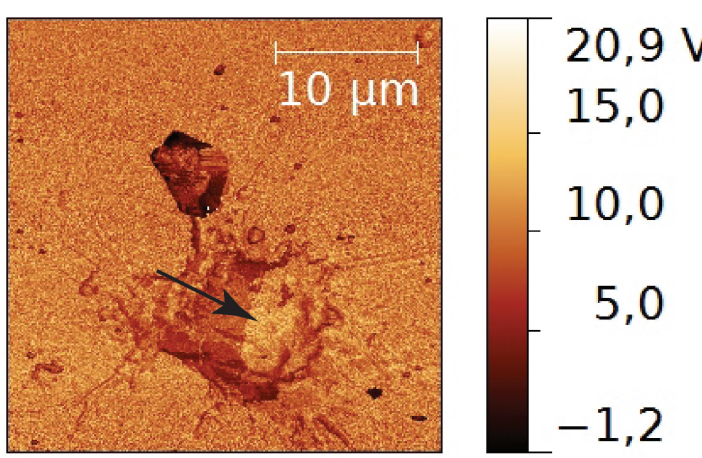

Fig. 6 (a) Topography of a microglia cell engulfed with NPs and (b) corresponding stiffness map obtained in Harmonix ${ }^{\mathrm{TM}}$ mode.

7 D. van der Zwaag, N. Vanparijs, S. Wijnands, R. De Rycke, B. G. De Geest and L. Albertazzi, ACS Appl. Mater. Interfaces, 2016, 8, 6391-6399.

8 A. E. Porter, M. Gass, K. Muller, J. N. Skepper, P. A. Midgley and M. Welland, Nat. Nanotechnol., 2007, 2, 713-717.

9 G. Binnig, C. F. Quate and C. Gerber, Phys. Rev. Lett., 1986, 56, 930-933.

10 U. Hartmann, Annu. Rev. Mater. Sci., 1999, 29, 53-87.

11 U. Rabe, J. Janser and W. Arnold, Rev. Sci. Instrum., 1996, 67, 3281-3293.

12 P. Girard, Nanotechnology, 2001, 12, 485-490.

13 A. Majumdar, Annu. Rev. Mater. Sci., 1999, 29, 505-585.

14 A. Noy, D. V. Vezenov and C. M. Lieber, Annu. Rev. Mater. Sci., 1997, 27, $381-$ 421.

15 G. Cordova, S. Attwood, R. Gaikwad, F. Gu and Z. Leonenko, Nano Biomed. Eng., 2014, 23, 31-39.

16 M. J. Cadena, R. Misiego, K. C. Smith, A. Avila, B. Pipes, R. Reifenberger and A. Raman, Nanotechnology, 2013, 24, 135706.

17 H. T. Thompson, F. Barroso-Bujans, J. G. Herrero, R. Reifenberger and A. Raman, Nanotechnology, 2013, 24, 135701.

18 Y. Zhang, M. Yang, M. Ozkan and C. S. Ozkan, Biotechnol. Prog., 2009, 25, $923-$ 928.

19 Z. Wang and A. Cuschieri, Int. J. Mol. Sci., 2013, 14, 9111-9125.

20 D. Passeri, C. Dong, M. Reggente, L. Angeloni, M. Barteri, F. A. Scaramuzzo, F. De Angelis, F. Marinelli, F. Antonelli, F. Rinaldi, C. Marianecci, M. Carafa, A. Sorbo, D. Sordi, I. W. C. E. Arends and M. Rossi, Biomatter, 2014, 4, e29507.

21 C. Roduit, S. Sekatski, G. Dietler, S. Catsicas, F. Lafont and S. Kasas, Biophys. J., 2009, 97, 674-677.

22 C. Roduit, G. Longo, I. Benmessaoud, A. Volterra, B. Saha, G. Dietler and S. Kasas, J. Mol. Recognit., 2012, 25, 241-246.

23 K. Yamanaka, H. Ogiso and O. V. Kolosov, Appl. Phys. Lett., 1994, 64, 178-180.

24 G. Shekhawat, A. Srivastava, S. Avasthy and V. P. Dravid, Appl. Phys. Lett., 2009 95, 263101.

25 G. S. Shekhawat and V. P. Dravid, Science, 2005, 310, 89-92.

26 A. C. Diebold, Science, 2005, 310, 61-62.

27 L. Tetard, A. Passian, K. T. Venmar, R. M. Lynch, B. H. Voy, G. Shekhawat, V. Dravid and T. Thundat, Nat. Nanotechnol., 2008, 3, 501-505.

28 L. Tetard, A. Passian, R. H. Farahi and T. Thundat, Ultramicroscopy, 2010, 110 586-591.

29 S. Amelio, A. V. Goldade, U. Rabe, V. Scherer, B. Bhushan and W. Arnold, Thin Solid Films, 2001, 392, 75-84.

30 U. Rabe, M. Kopycinska, S. Hirsekorn, J. Muñoz Saldaña, G. A. Schneider and W. Arnold, J. Phys. D, 2002, 35, 2621-2635.

31 D. Passeri, A. Bettucci, M. Germano, M. Rossi, A. Alippi, V. Sessa, A. Fiori, 
E. Tamburri and M. L. Terranova, Appl. Phys. Lett., 2006, 88, 121910.

32 D. Passeri, M. Reggente, M. Rossi, S. Nunziante Cesaro, V. Guglielmotti, J. J. Vlassak, A. M. De Francesco, R. Scarpelli, M. Hatipoğlu and D. Ajò, Eur. J. Mineral., 2016, 28, 273-283.

33 J. P. Killgore, D. G. Yablon, A. H. Tsou, A. Gannepalli, P. A. Yuya, J. A. Turner, R. Proksch and D. C. Hurley, Langmuir, 2011, 27, 13983-13987.

34 D. G. Yablon, A. Gannepalli, R. Proksch, J. Killgore, D. C. Hurley, J. Grabowski and A. H. Tsou, Macromolecules, 2012, 45, 4363-4370.

35 D. C. Hurley, S. E. Campbell, J. P. Killgore, L. M. Cox and Y. Ding, Macromolecules, 2013, 46, 9396-9402.

36 A. Ebert, B. R. Tittmann, J. Du and W. Scheuchenzuber, Ultrasound Med. Biol., 2006, 32, 1687-1702.

37 D. Passeri, M. Rossi, A. Alippi, A. Bettucci, M. L. Terranova, E. Tamburri and F. Toschi, Physica E, 2008, 40, 2419-2424.

38 M. Reggente, M. Rossi, L. Angeloni, E. Tamburri, M. Lucci, I. Davoli, M. L. Terranova and D. Passeri, JOM, 2015, 67, 849-857.

39 M. Natali, D. Passeri, M. Reggente, E. Tamburri, M. L. Terranova and M. Rossi, AIP Conf. Proc., 2016, 1749, 020008.

40 Z. Parlak and F. L. Degertekin, J. Appl. Phys., 2008, 103, 114910.

41 J. P. Killgore, J. Y. Kelly, C. M. Stafford, M. J. Fasolka and D. C. Hurley, Nanotechnology, 2011, 22, 175706.

42 R. Massart, IEEE T. Magn., 1981, 17, 1247-1248.

43 F. De Angelis, G. Berardi, F. A. Scaramuzzo, M. Liberatore and M. Barteri, Int. J. Nanotechnol., 2016, 13, 659-666.

44 M. Jaafar, O. Iglesias-Freire, L. Serrano-Ramón, M. R. Ibarra, J. M. de Teresa and A. Asenjo, Beilstein J. Nanotechnol., 2011, 2, 552-560.
45 L. Angeloni, D. Passeri, M. Reggente, D. Mantovani and M. Rossi, Sci. Rep., 2016 , 6, 26293.

46 D. Passeri, A. Bettucci, M. Germano, M. Rossi, A. Alippi, A. Fiori, E. Tamburri, M. L. Terranova and J. J. Vlassak, Microelectr. Eng., 2007, 84, 490-494.

47 J. P. Killgore and D. C. Hurley, Nanotechnology, 2012, 23, 055702.

48 O. Sahin, S. Magonov, C. Su, C. F. Quate and O. Solgaard, Nat. Nanotechnol., 2007, 2, 507-514.

49 O. Sahin and N. Erina, Nanotechnology, 2008, 19, 445717.

50 P. Schön, K. Bagdi, K. Molnár, P. Markus, B. Pukánszky and G. J. Vancso, Eur Polym. J., 2011, 47, 692-698.

51 M. E. Dokukin and I. Sokolov, Langmuir, 2012, 28, 16060-16071.

52 D. Passeri, M. Rossi, E. Tamburri and M. L. Terranova, Anal. Bioanal. Chem., 2013, 405, 1463-1478.

53 D. Passeri, A. Biagioni, M. Rossi, E. Tamburri and M. L. Terranova, Eur. Polym. J., 2013, 49, 991-998.

54 K. M. Leung, G. Wanger, Q. Guo, Y. Gorby, G. Southam, W. M. Laue and J. Yang, Soft Matter, 2011, 7, 6617-6621.

55 K. Sweers, K. van der Werf, M. Bennink and V. Subramaniam, Nanoscale Res. Lett., 2011, 6, 270.

56 M. Rossi, F. Cubadda, L. Dini, M. L. Terranova, F. Aureli, A. Sorbo and D. Passeri, Trends Food Sci. Tech., 2014, 40, 127-148.

57 D. Passeri, E. Tamburri, M. L. Terranova and M. Rossi, Nanoscale, 2015, 7 , 14358-14367.

58 R. B. King, Int. J. Solids Structures, 1987, 23, 1657-1664. 


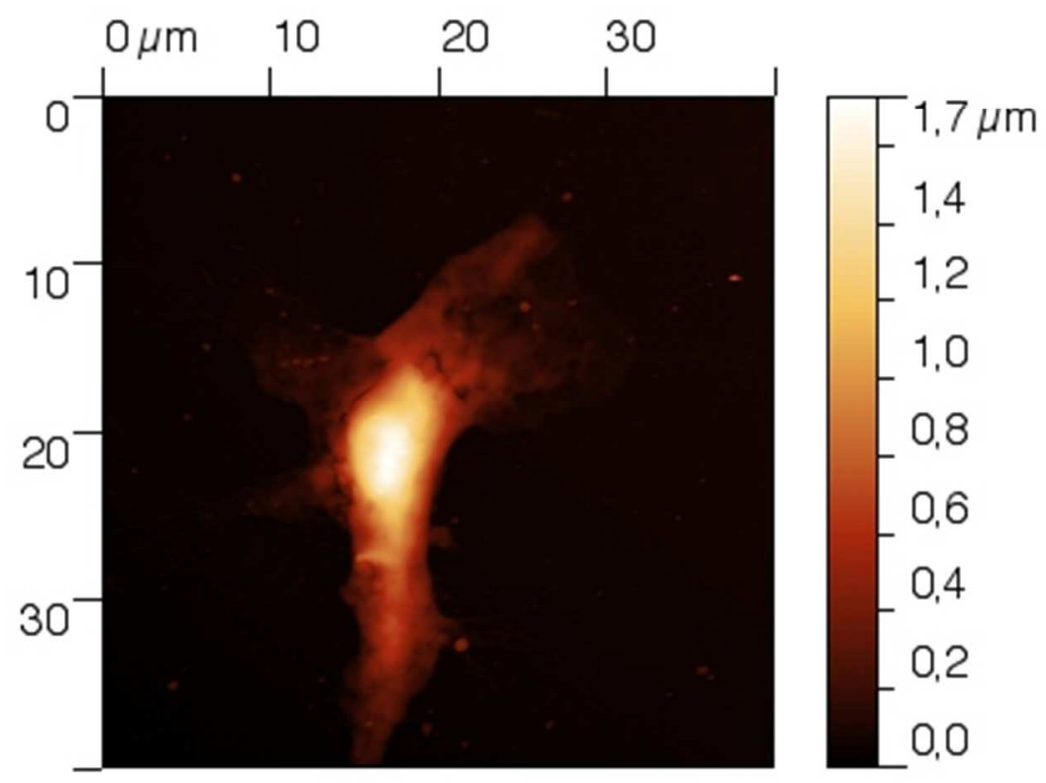

Figure 1

$155 \times 118 \mathrm{~mm}(72 \times 72$ DPI $)$ 


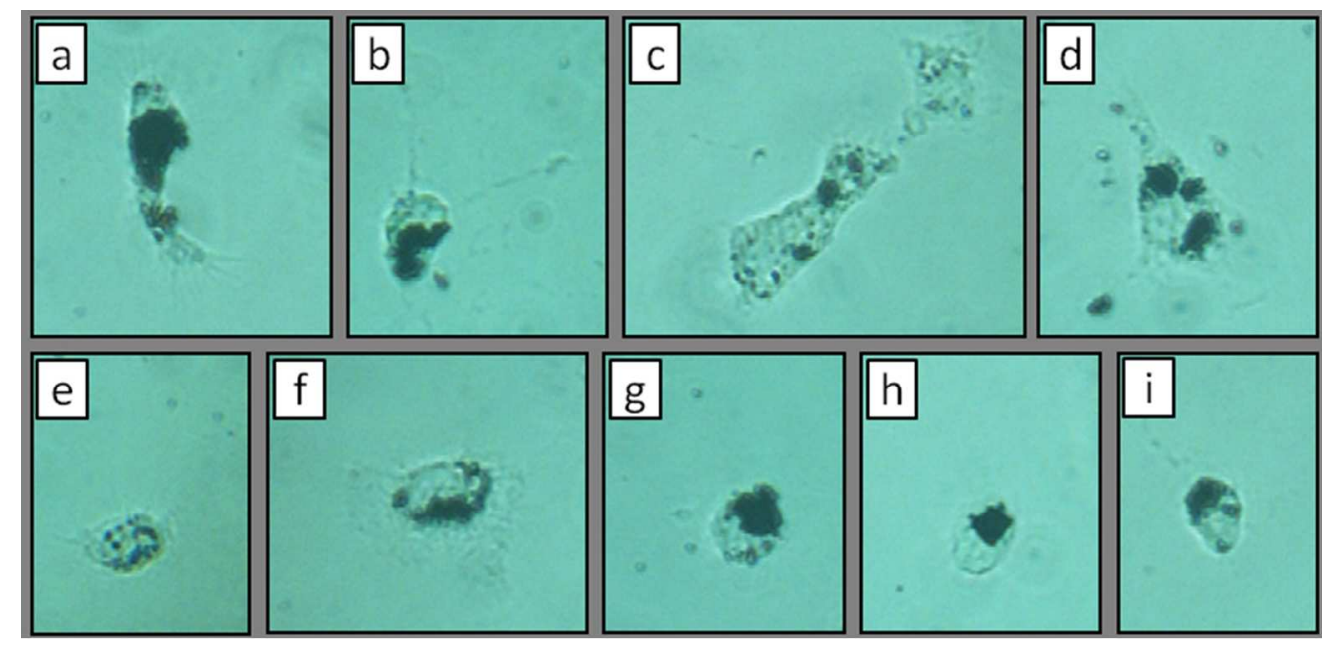

Figure 2 


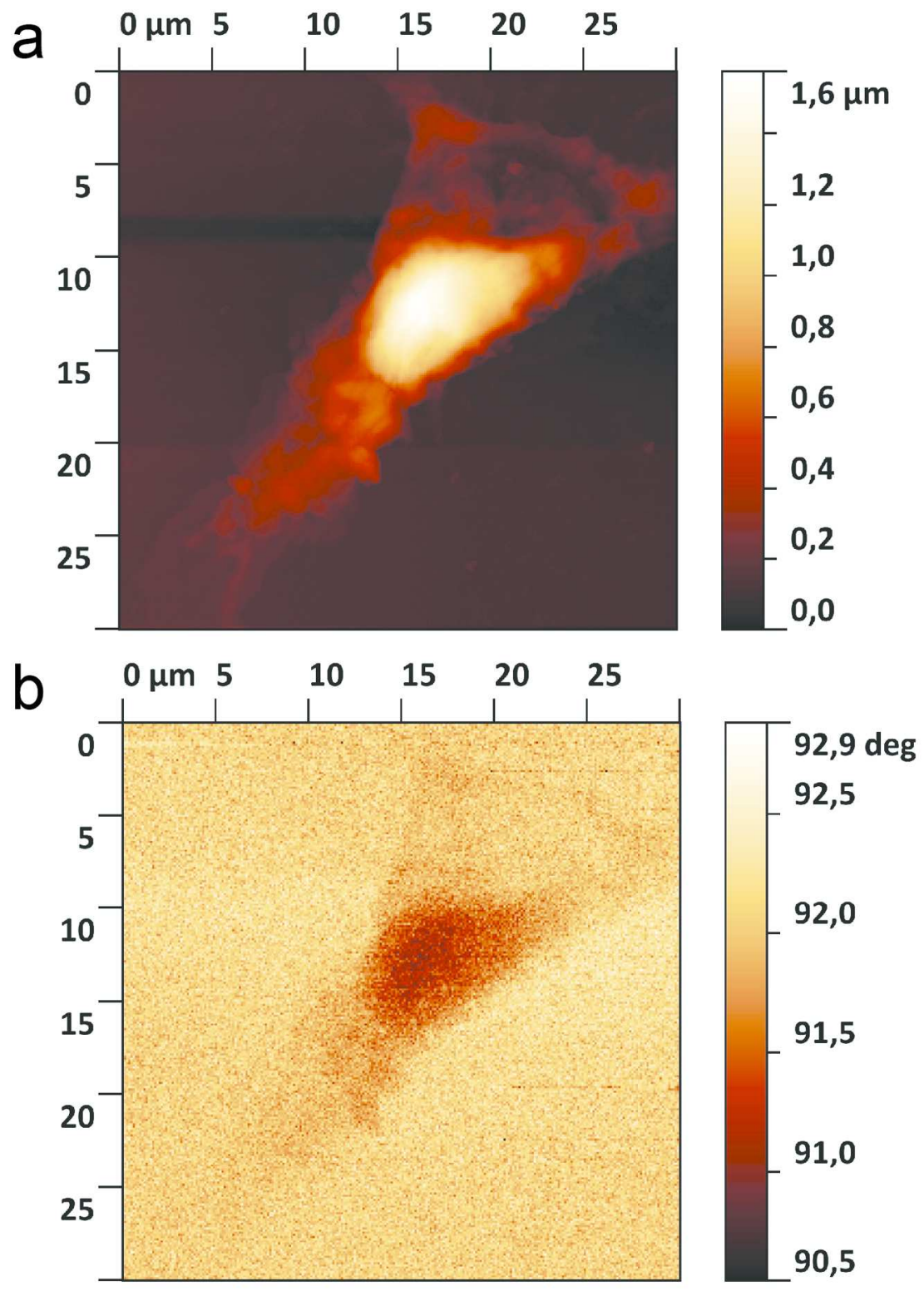

Figure 3

$133 \times 190 \mathrm{~mm}(300 \times 300$ DPI $)$ 

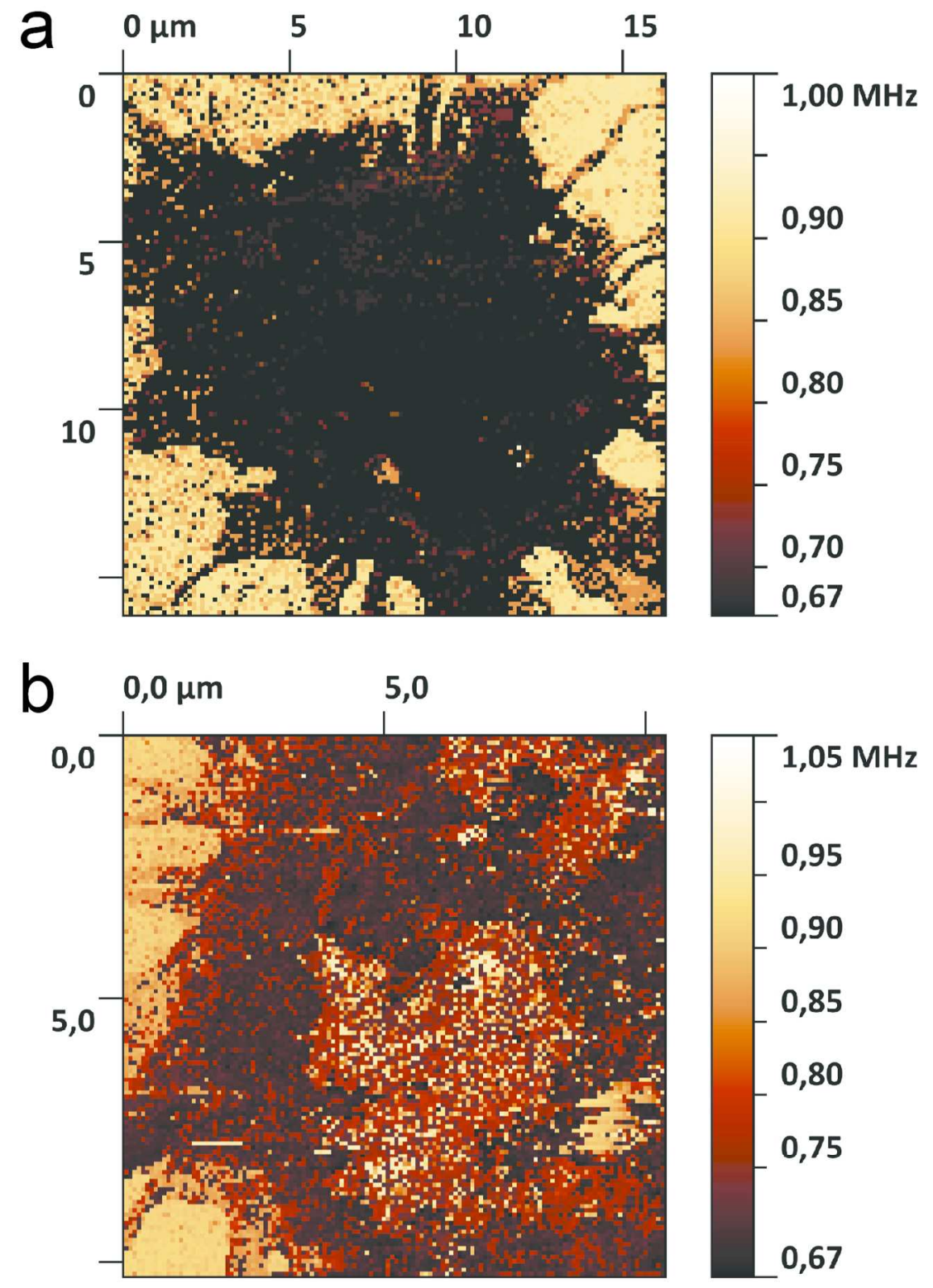

Figure 4

$121 \times 172 \mathrm{~mm}(300 \times 300 \mathrm{DPI})$ 


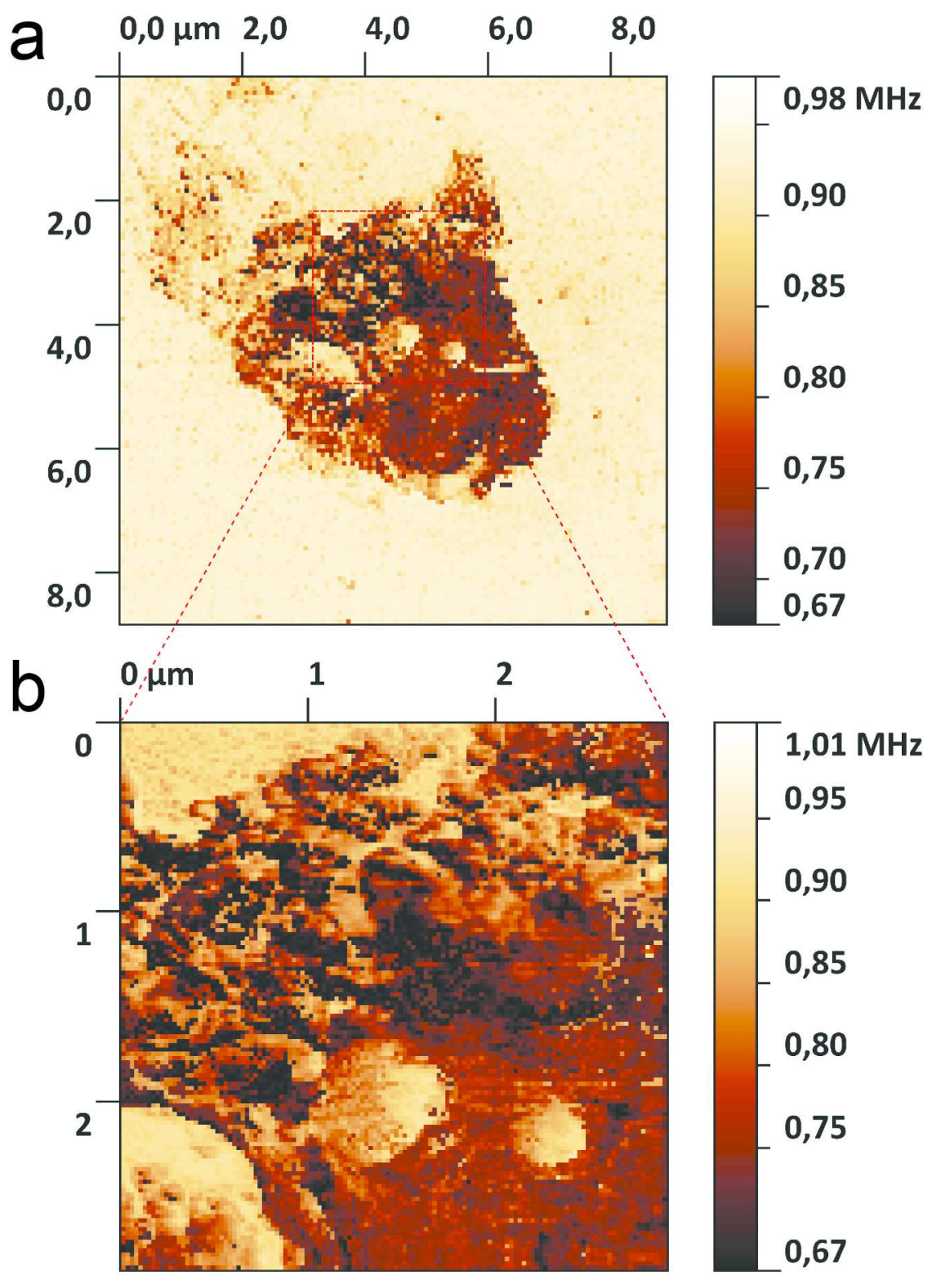

Figure 5

$165 \times 229 \mathrm{~mm}(300 \times 300$ DPI $)$ 


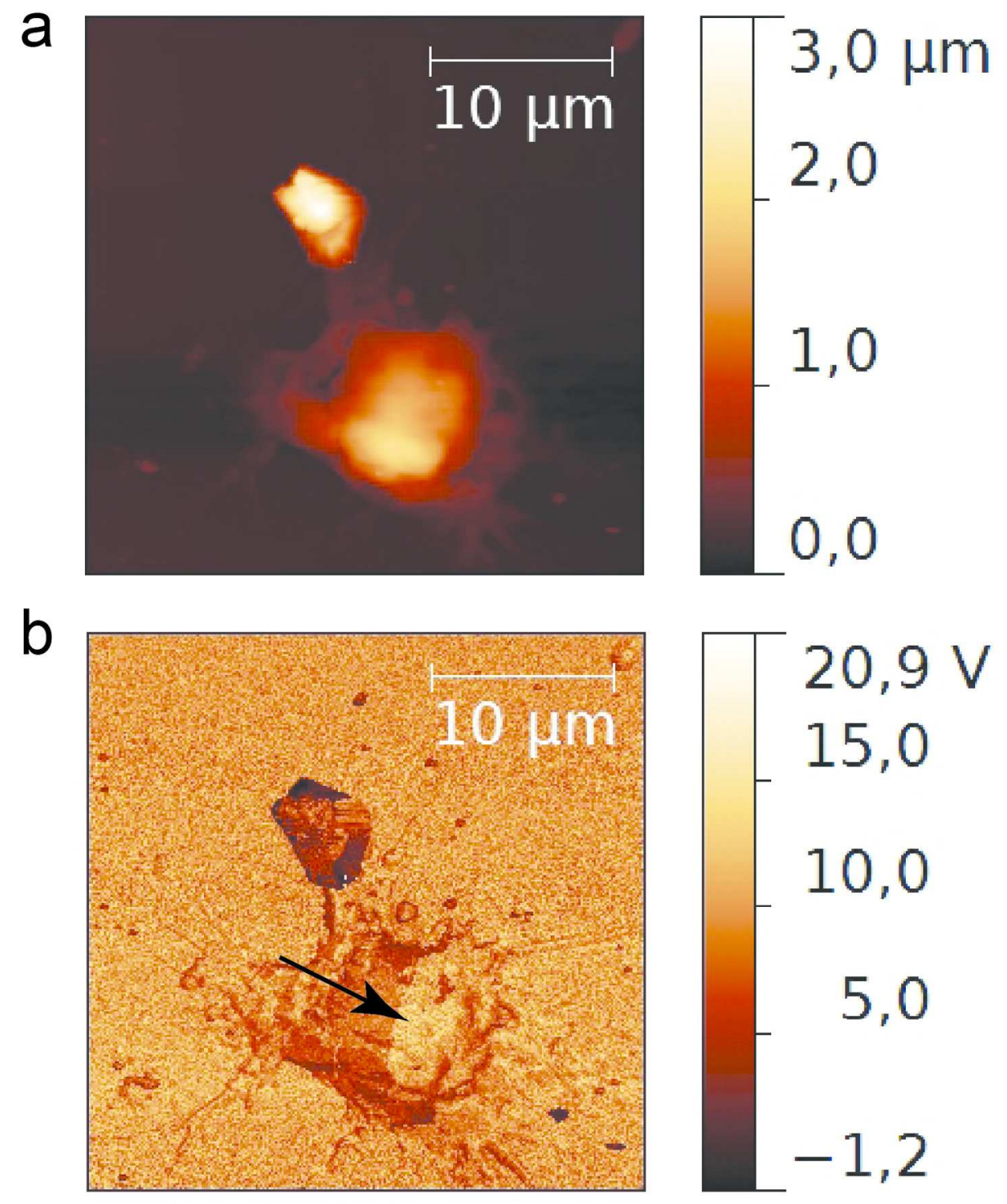

Figure 6

149x186mm (300 x 300 DPI) 